Research Article

\title{
Green Synthesis of Silver Nanoparticles Using Cosmos Sulphureus and Evaluation of Their Antimicrobial and Antioxidant Properties
}

\author{
Rajkumari Malaka ${ }^{1}$, Jerusha A Hema ${ }^{1}$, Narayanan P Muthukumarasamy ${ }^{1}$ \\ Akilandeswari Sambandam ${ }^{2}$, Subakanmani Subramanian ${ }^{1}$, Murugan Sevanan ${ }^{1}$ \\ ${ }^{1}$ Department of Biotechnology, School of Biotechnology and Health Sciences, Karunya University, Karunya Nagar, Coimbatore \\ 641114, India \\ ${ }^{2}$ Department of Physics, Faculty of Science, Annamalai University, Annamalai Nagar, Chidambaram 608002, India \\ MCorresponding author: micromurugans@gmail.com
}

Received: Aug. 24, 2015; Accepted: Dec. 28, 2015; Published: Jan. 14, 2016.

Citation: Rajkumari Malaka, Jerusha A Hema, Narayanan P Muthukumarasamy, Akilandeswari Sambandam, Subakanmani Subramanian and Murugan Sevanan. Green Synthesis of Silver Nanoparticles Using Cosmos Sulphureus and Evaluation of Their Antimicrobial and Antioxidant Properties. Nano Biomed. Eng. 2015, 7(4), 160-168.

DOI: $10.5101 /$ nbe.v7i4.p160-168.

\begin{abstract}
The present study intended to synthesize, characterize silver nanoparticles (AgNPs) using Cosmos sulphureus (Cs) aqueous leaf extract and their antimicrobial and antioxidant activities. The UV-vis spectrophotometer exhibited an absorbance peak around 430-440 nm with color intensity from yellow to reddish brown after 4 days. SEM and EDX analysis showed AgNPs were pure and polydispersed and the size were ranging from 55 to $80 \mathrm{~nm}$. XRD revealed that the nanoparticles were cubic and face centered in shape and the biogenic CsAgNPs showed effective potent antimicrobial and antioxidant activities. This study may serve as a tool for analyzing the controlled release of synthesized AgNPs, which have prospective role in the field of disease management particularly with respect to resistant pathogenic microorganisms.
\end{abstract}

Keywords: Cosmos sulphureus; silver nanoparticles; Antimicrobial, Antioxidant

\section{Introduction}

In the recent years, the use of metal nanoparticles have gained greater advantage due to their vast applications in the field of medicine, biology, physics, chemistry and material sciences [1]. Among them, silver nanoparticles (AgNPs) have attracted because of their individual properties, like good electrical conductivity, chemical stability, catalytic and antibacterial activity [2]. Application of silver nanoparticles includes clothing [3], food industry [4], agriculture [5], textile industry [6], water treatment [7] and therapeutic agents for diagnosing chronic diseases [8]. Silver is a better substitute compared to the other metals as it has very less or no toxic effect and also they are widely used as bactericide in catheters, burn wounds and dental work $[9,10]$.

Biogenic or biological approach of nanoparticle synthesis have received greater attention over physical and chemical synthesis, as it is non-toxic, nonhazardous, cost effective and eco-friendly [11, 12]. Biosynthesis of silver nanoparticles has advantages 
like slower kinetics, better manipulation and control over crystal growth and excellent stability [13]. But, microbial mediated synthesis of AgNPs is not industrially achievable as they need high maintenance of aseptic conditions. Therefore, the use of plant extract for the synthesis of AgNPs is comparatively advantageous over other biological methods [14]. $C$. sulphureus is a member of the family Compositae, commonly referred to as yellow cosmos and Klondike cosmos spread throughout India, Florida, Southern United States and South America [15]. They are generally used for improving blood circulation, antiaging agent, reducing body heat, strengthening bone marrow (because of its high calcium content) and to treat infections associated with pathogenic microorganisms [16]. Recently, Bindurani et al. [16] isolated butein from C. sulphureus which is a powerful antioxidant against lipid and LDL (Low density lipoprotein) and also exhibit anti-inflammatory activity, aromatase inhibition [17] and cyclooxygenase inhibition [18]. In the present study, we have demonstrated the biogenic synthesis of AgNPs by reduction of aqueous silver nitrate using $C s$ aqueous leaf extract. Additionally, we have also examined the antimicrobial and antioxidant activity of synthesized AgNPs.

\section{Materials and Methods}

\section{Materials}

Silver nitrate, $\mathrm{AgNO}_{3}$, Ethyl alcohol, $\mathrm{C}_{2} \mathrm{H}_{6} \mathrm{O}$, Carbinol, $\mathrm{CH}_{4} \mathrm{O}$, and 2,2-dipheny-1-picrylhydrazyl $(\mathrm{DPPH}), \mathrm{C}_{18} \mathrm{H}_{12} \mathrm{~N}_{5} \mathrm{O}_{6}$ were of analytical grade and used without further purification. C. sulphureus leaves were collected from Karunya University campus, Coimbatore, South India. Plant species authentication was done at Botanical Survey of India (BSI), Coimbatore, South India (Ref No. BSI/ $\mathrm{SRC} / 5 / 23 / 2014-15 / 193)$. The clinical isolates such as Staphylococcus aureus, Salmonella typhi, Escherichia coli, Pseudomonas aeruginosa, Klebsiella pneumoniae, Candida albicans, Fusarium sp. and Rhizopus oryza were obtained from Microbiology laboratory, Kovai Medical Center and Hospital (KMCH), Coimbatore, South India

\section{Preparation of aqueous leaf extract}

The leaves of C. sulphureus was washed thoroughly with distilled water several times to remove the dust particles. Ten grams of chopped leaves were boiled with $200 \mathrm{ml}$ of deionized water for $15 \mathrm{~min}$ at $60^{\circ} \mathrm{C}$ to obtain the extract. The crude extract was then filtered using Whatmann No. 1 filter paper (Maidstone U.K) and stored at room temperature for further experiments.

\section{Synthesis of Silver Nanoparticles (AgNPs)}

Exactly $10 \mathrm{~mL}$ of the aqueous leaf extract was added to $90 \mathrm{~mL}$ of $1 \mathrm{mM} \mathrm{AgNO}_{3}$ solution and incubated until color change. The solution turned to brown color indicating the formation of $C$. sulphureus silver nanoparticles (CsAgNPs). The mixture was boiled at $80^{\circ} \mathrm{C}$ for $10 \mathrm{~min}$ with continuous stirring and filtered through Whatmann filter paper No.1 (Maidstone, UK). The filtrate was lyophilized at Defense Research and Development Organization (DRDO), Bharathiar University, Coimbatore for further studies.

\section{Characterization of Synthesized Silver Nanoparticles}

The synthesized silver nanoparticles were initially confirmed by UV-Vis analysis at different time intervals and the absorption maxima was scanned between 300-700 nm wavelengths using Hitachi U2910 spectrophotometer. The particle size and zeta potential of the synthesized $C s \mathrm{AgNPs}$ were analyzed using Malvern particle size analyzer using Dynamic Light Scattering (DLS) technique. Also, the morphological, structural and chemical composition of CsAgNPs were analyzed by SEM-EDX (JEO-JSM 6390, Japan) and XRD (XRD-6000 X-ray diffractometer, Shimadzu, Japan). The functional groups present in $C s \mathrm{AgNPs}$ causing the reduction was recorded by FTIR (Shimadzu 8400S, Japan).

\section{Determination of Antimicrobial activity of CsAgNPs}

In vitro antimicrobial activity of the synthesized CsAgNPs was determined by agar well diffusion method [19]. Fresh overnight inoculums $\left(100 \mu 1-10^{5}\right.$ $-10^{6} \mathrm{CFU} / \mathrm{ml}$ ) of each clinical isolates were swabbed uniformly on Mueller Hinton (MH) agar plates using sterile cotton swab. Then three wells of $6 \mathrm{~mm}$ diameter were made using sterile well borer. Hundred microliter of $C s A g N P s$ solutions with various concentrations (75, 100 and $150 \mu \mathrm{g} / \mathrm{ml}$ ) was poured into the corresponding well. The plates were incubated at $37^{\circ} \mathrm{C}$ for 24 and $48 \mathrm{~h}$ for the bacterial and fungal cultures, respectively. 
The diameter of inhibition zone was measured after incubation. Deionized water was used as a negative control and the antibiotics Ofloxacin (5 $\mu \mathrm{g} / \mathrm{disc})$, Tobramycin (10 $\mu \mathrm{g} /$ disc), Ciprofloxacin $(30 \mu \mathrm{g} / \mathrm{disc})$ and Gentamycin $(30 \mu \mathrm{g} / \mathrm{disc})$ were used as positive controls. Triplicates were maintained and average values were calculated.

\section{In vitro antioxidant assays DPPH Free Radical Scavenging Assay}

The DPPH free radical scavenging assay was conducted based on the method of Chang et al. [20]. One milliliter of $0.1 \mathrm{mM}$ DPPH (in ethanol) was added to different concentrations $(50,100,150$ and $200 \mu \mathrm{g} /$ ml) of Cs aqueous leaf extract and CsAgNPs. The reaction mixtures were incubated in the dark for 30 minutes. The absorbance at $517 \mathrm{~nm}$ was measured against a blank (ethanol). Ascorbic acid was used as the standard. The lower absorbance of the reaction mixture indicated a higher percentage of scavenging activity. The percentage of inhibition or scavenging of free radicals was determined by the following formula: $\%$ inhibition $=$

[ $\{$ Control OD-Sample OD $\} /$ Control OD] x 100

\section{Results and Discussion}

\section{UV-Vis spectral Studies}

The color change of the $C s$ aqueous leaf extract was noticed visually when the extract was incubated with the $\mathrm{AgNO}_{3}$ solution (Fig. 1). Absorption spectrum revealed a peak between 430-440 nm (Fig. 2) suggesting bioreduction of silver nitrate into silver nanoparticles. The color of the extract changed from yellow to reddish brown after 4 days of incubation and leaf extract without $\mathrm{AgNO}_{3}$ did not show any color change. Currently, the biogenic synthesis of nanoparticles is the most effective technology towards the development of biologically inspired processes. The present study emphasizes the use of medicinal plants for the synthesis of silver nanoparticles with their potent antimicrobial and antioxidant effects. The reduction of silver ions and the formation of stable nanoparticles occurred on $4^{\text {th }}$ day to synthesize silver nanoparticles. The interaction between nanoparticles and biomolecules have been studied through several analysis and found that, various terpenoids or alkaloids present in the plant extracts caused the bioreduction
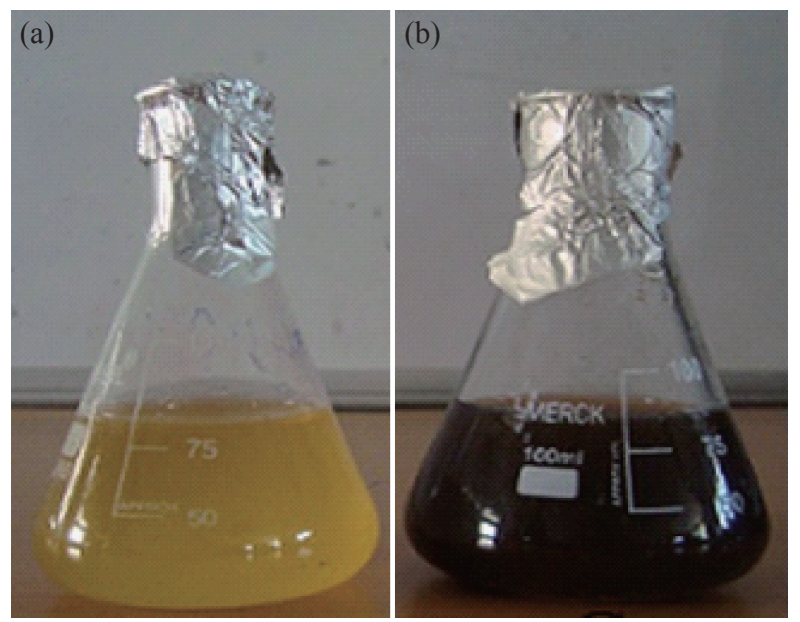

Fig. 1 (a) C. sulphureus leaf extract, (b) Color change after synthesized $C s$ AgNPs.

of metal ions and stabilization of AgNPs [13]. The plant assisted reduction due to phytochemicals are considered to be the main mechanism for the synthesis of nanoparticles. The phytochemicals involved are terpenoids, flavones, ketones, aldehydes, amides, and carboxylic acids which are soul responsible for the direct reduction of ions and formation of silver nanoparticles [21, 22]. Although, the each plant mechanism will be varied as the phytochemicals involved are different, but the main mechanism is reduction of ions.

The surface plasmon band in the silver nanoparticles solution remains close to $440 \mathrm{~nm}$ throughout the reaction period indicating that the particles are dispersed in the aqueous solution, with possible evidence for aggregation. Earlier studies have shown that absorbance at around 400-500 $\mathrm{nm}$ for silver is a characteristic of these metal particles [23, 24]. Moreover, Y-yuan et al. [25] showed absorbance of AgNPs synthesized by three different leaf extracts are at 447, 445 and $482 \mathrm{~nm}$. Also, AgNPs capped with L-cysteine showed absorption maximum at $440 \mathrm{~nm}$ with 1:5 ratio [26]. This showed that present study results were highly significance with previous reports.

\section{Particle size and Zeta potential measurements}

Particle size determination of the synthesized AgNPs exhibited different size ranging from 50 to 70 nm which was found to be polydisperse in nature (Fig. 3 ). The average diameter of the particles was found to be $70 \mathrm{~nm}$. Meanwhile, zeta potential measurement of synthesized $C s A g N P s$ showed the surface charges of the particles and exhibited a value of $-22.7 \mathrm{mV}$ (Fig. 


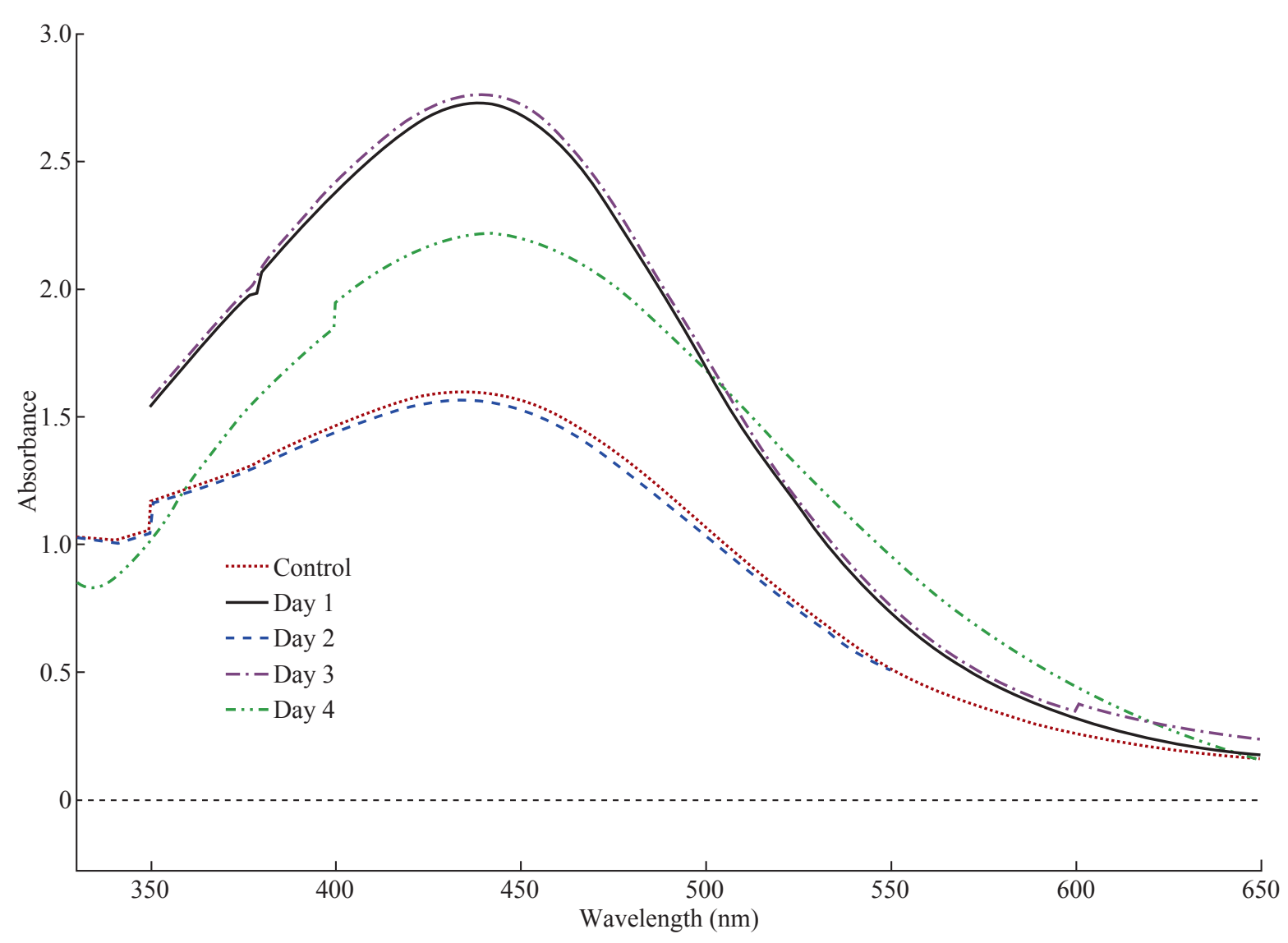

Fig. 2 The UV-vis absorption spectrum of silver nitrate $(1 \mathrm{mM})$ as control and synthesized CsAgNPs at different time intervals (days).

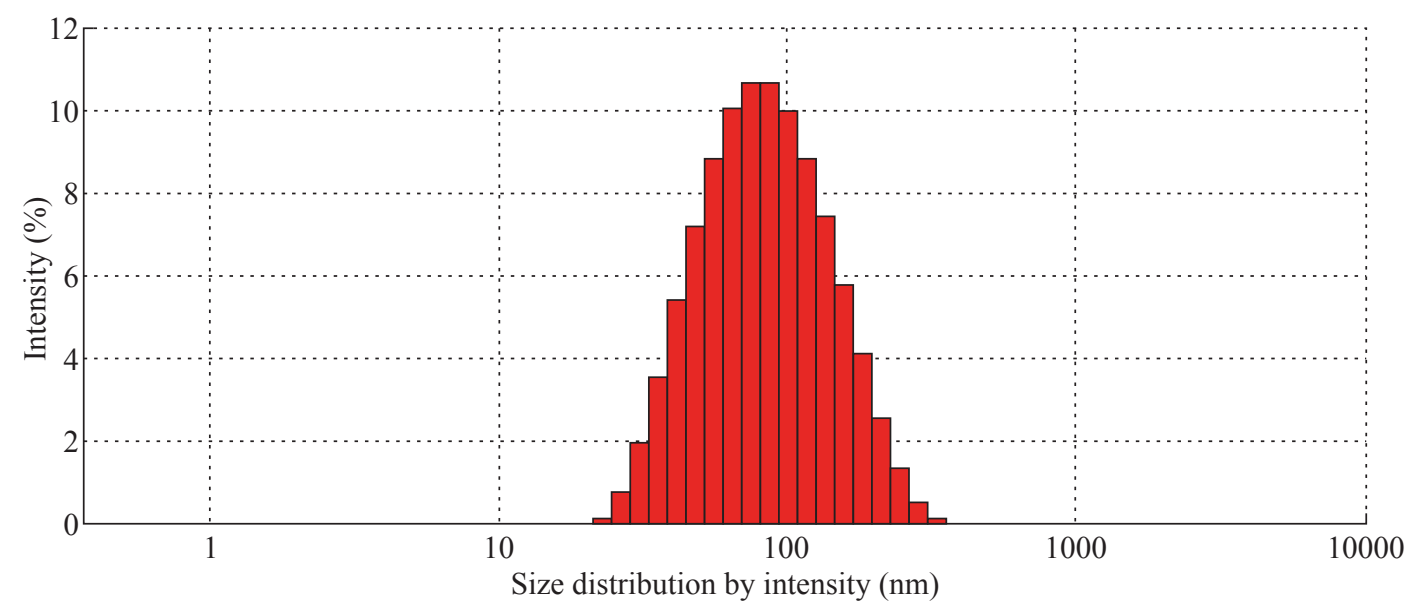

Fig. 3 Particle size distribution curve for synthesized CsAgNPs.

4), suggesting higher stability of AgNPs. The large negative potential value could be due to the capping of polyphenolic constituents present in the $C s$ aqueous leaf extract. The particle size of AgNPs synthesized from Malus domestica was observed between 50$300 \mathrm{~nm}$ with average of $150 \mathrm{~nm}$ whereas in the current study, it was $70 \mathrm{~nm}$. The reason for large size distribution was due to time fluctuation in the intensity of light scattering by autocorrelator, which determines the signal autocorrelation function of nanoparticles [27]. Moreover, the zeta potential of Achillea biebersteinii AgNPs showed potential range between -20 to $-40 \mathrm{mV}$ [28] and AgNPs from Lantana camara leaf extract found between -25 to $-50 \mathrm{mV}$ [29] which is in consistent with AgNPs from C. sulphureus (-22.7 to $-40 \mathrm{mV}$ ). The high electric charge on the surface of nanoparticles determines high absolute zeta potential which was due to capping of bio-organic compounds 


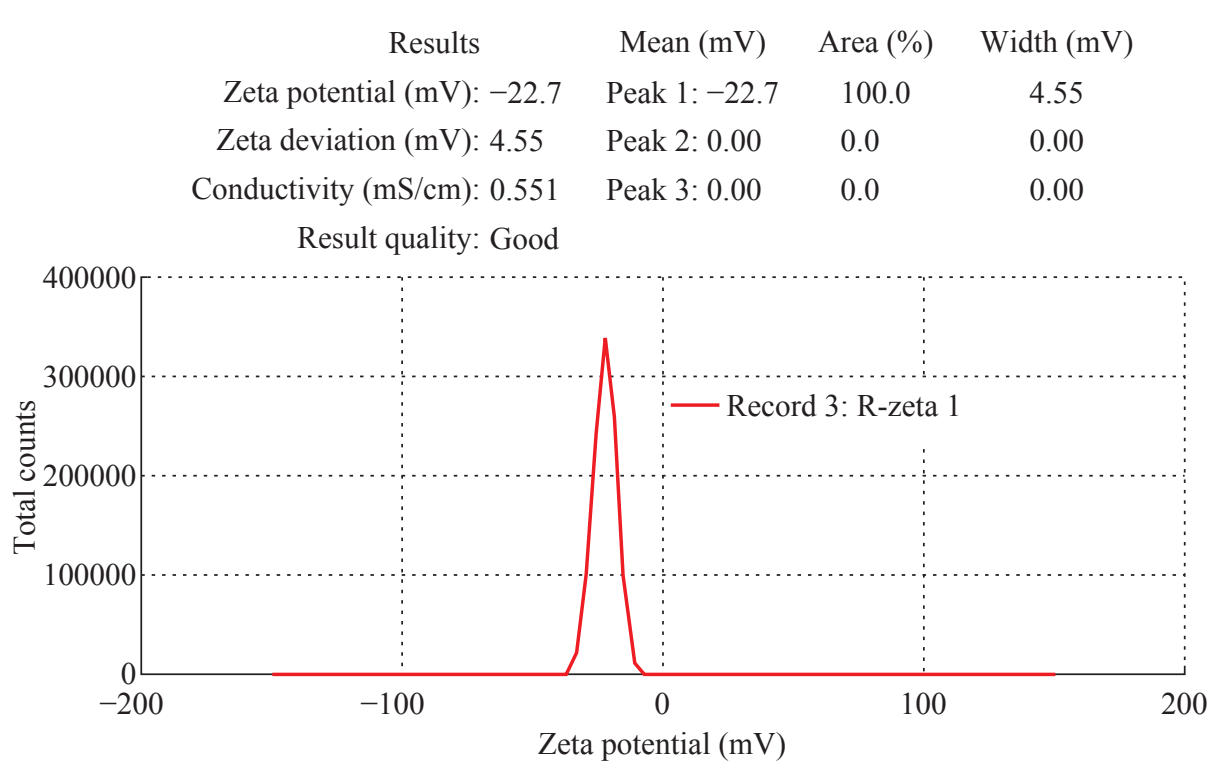

Fig. 4 Zeta potential measurement of synthesized CsAgNPs.

present in the plant leaf extract [30].

\section{Characterization studies}

$\mathrm{X}$-ray diffraction pattern of the biosynthesized CsAgNPs were confirmed by three characteristic peaks of $32.19^{\circ}, 29.37^{\circ}$ and $27.8^{\circ}$ in the whole spectrum of $2 \theta$ value ranging from 10 to 90 (Fig. 5). SEM analysis was carried out to understand the topology and the size of the synthesized AgNPs, which showed the synthesis of higher density polydispersed spherical AgNPs of various sizes that ranged from 55-80 $\mathrm{nm}$. Most of the nanoparticles aggregated and only a few of them were scattered, as observed like a flower shaped under SEM (Fig. 6(a)). The EDX spectrum of synthesized silver nanoparticles (Fig. 6(b)) clearly exhibited the absence of elemental nitrogen and oxygen peaks and the

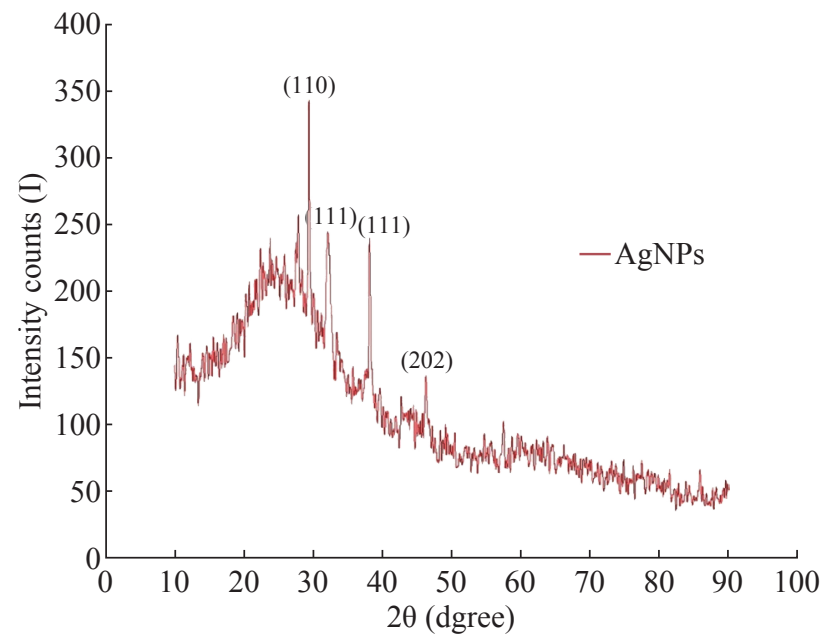

Fig. 5 XRD pattern of CsAgNPs.

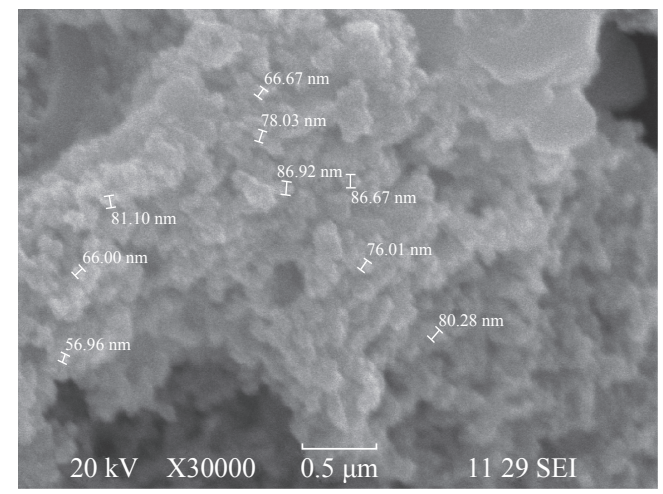

(a)

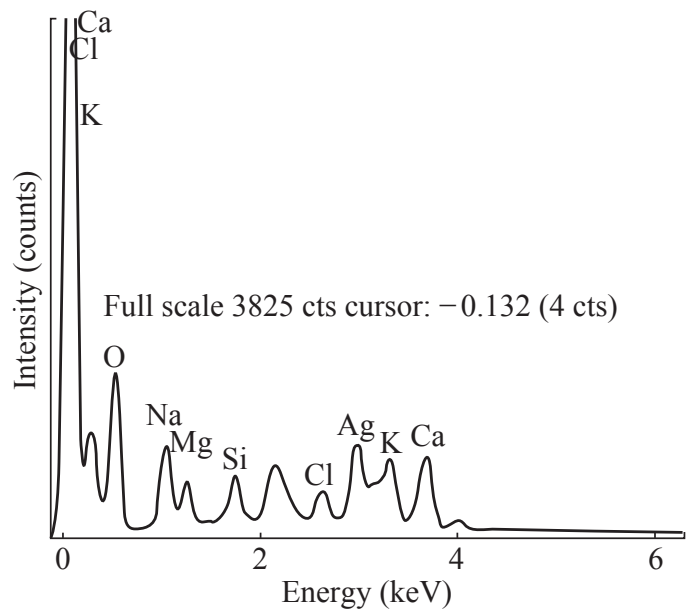

(b)

Fig. 6 (a) The SEM image of CsAgNPs, (b) The EDX image of CsAgNPs.

presence of elemental silver metal. The sharp peak of silver strongly confirmed the reduction of silver nitrate to silver nanoparticles.

FTIR measurements of both the aqueous leaf extract 


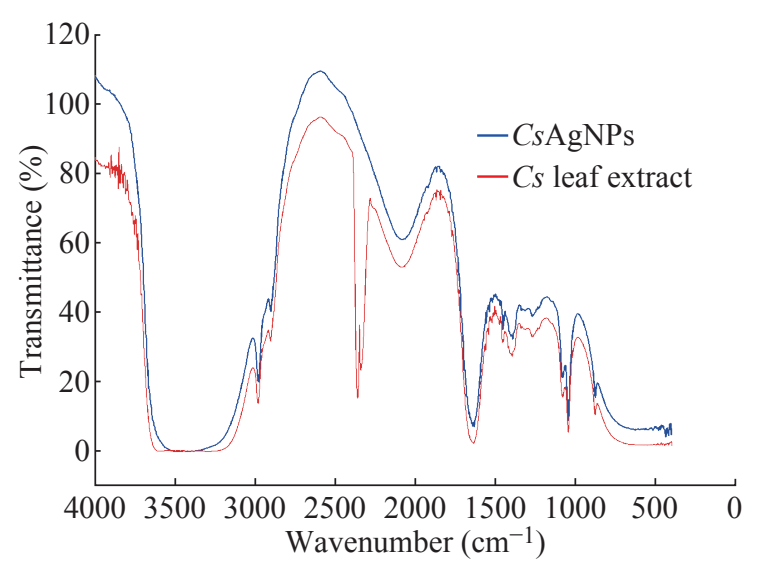

Fig. 7 (a) The FTIR spectrum of $C s$ leaf extract and $C s$ AgNPs.

and $C s A g N P s$ were carried out to identify the possible metabolites responsible for the reduction of $\mathrm{Ag}^{+}$ions and capping of the bioreduced silver nanoparticles synthesized by the leaf extract. The major peaks of silver nanoparticles (Fig. 7) were found to be 1643.35 ( $-\mathrm{C}==\mathrm{C}-\mathrm{-}$ stretch of alkenes), $2980.02(\mathrm{C}--\mathrm{H}$ stretch of alkanes) and $3421.72 \mathrm{~cm}^{-1}(\mathrm{O}-\mathrm{H}$ stretch, H-bonded of alcohols and phenols). Similarly, Cs aqueous leaf extract showed peaks at 1637.56, 2981.95 and 3748.2 which clearly indicates that reduction occurs at $-\mathrm{C}=\mathrm{C}-\mathrm{C}, \mathrm{C}-\mathrm{H}$ stretch of alkenes and alkanes. But, increase in peak was observed for Cs aqueous leaf extract represent high activities of alcohols and phenols during synthesis of silver nanoparticles.

Marimuthu et al. [31], reported average silver nanoparticle size of $35-55 \mathrm{~nm}$ from Gelidiella acerosa leaf extract but in the present study, it was found to be $55-80 \mathrm{~nm}$. Moreover, the shape of synthesized nanoparticles were visualized as spherical [32], whereas in the current study, it was flower shaped which proved the uniqueness of the synthesized nanoparticles. The optical absorption band of EDX peaks were in the range of 3 to $4 \mathrm{keV}$ which is typical for absorption of metallic silver nanoparticles [33]. The weight percentage of silver obtained for Cs AgNPs was found to be $14.45 \%$ which is in line with the previous studies by Ponarulselvam et al. [32] where EDX spectra of $C$. roseus nanoparticles showed silver weightage of 20.16 and $16.41 \%$. The XRD pattern of the present study showed mixed phases like cubic and face-centerd cubic of silver nanoparticles. The average particle shape of the nanoparticles was derived from the FWHM of the corresponding peak (111), (011), (101) and (110) planes with cubic $\left(2 \theta=32.19^{\circ}\right.$, and), face-centered cubic $\left(2 \theta=29.37^{\circ}\right)$ structures of the CsAgNPs and $27.8^{\circ}$ was due to the presence of organic compounds in the extracts $[34,35]$ This findings clearly specified that the silver nanoparticles formed by the reduction of $\mathrm{Ag}^{+}$ions by the $C s$ aqueous leaf extract are crystalline in nature. This was well supported by a study on synthesis of silver nanoparticles from leaf extract of C. roseus [32]. While comparing the FTIR peaks, the aqueous extract of $C s$ and $C s A g N P s$, there is a decrease in the intensity of peaks and also withdrawal of peaks at major spectrum were observed. The reason may be that the hydroxyl groups of phenols and amide groups of proteins forming a layer to the nanoparticles and acting as capping agents to prevent agglomeration and providing stability to the medium [36, 37]

\section{Antimicrobial studies}

The antibacterial activity of the synthesized CsAgNPs in different concentrations ( 75 to $150 \mu \mathrm{l}$ ) was quantitatively assessed on the basis of zone of inhibition (Table 1). According to Clinical and Laboratory Standards Institute, guidelines, the

Table 1 Antimicrobial activity of $C s$ AgNPs

\begin{tabular}{|c|c|c|c|}
\hline \multirow{2}{*}{ Clinical isolates } & \multicolumn{3}{|c|}{ CsAgNPs $(\mu \mathrm{g} / \mathrm{ml}) /$ Zone of inhibition $(\mathrm{mm})$} \\
\hline & $75 \mu \mathrm{g} / \mathrm{ml}$ & $100 \mu \mathrm{g} / \mathrm{ml}$ & $150 \mu \mathrm{g} / \mathrm{ml}$ \\
\hline E. coli & $10.4 \pm 0.5$ & $11.7 \pm 0.8$ & $14.3 \pm 0.3$ \\
\hline S. aureus & $8.4 \pm 0.5$ & $9.5 \pm 0.5$ & $11.2 \pm 0.8$ \\
\hline K. pneumoniae & $12.8 \pm 0.6$ & $13.5 \pm 0.5$ & $15.4 \pm 0.3$ \\
\hline$P$. aeruginosa & $9.5 \pm 0.5$ & $10.5 \pm 0.5$ & $11.5 \pm 0.5$ \\
\hline S. typhi & $8.3 \pm 0.6$ & $9.4 \pm 0.8$ & $10.5 \pm 0.5$ \\
\hline C. albicans & $12.7 \pm 0.5$ & $13.2 \pm 0.4$ & $14.2 \pm 0.3$ \\
\hline Fusarium $s p$ & $8.2 \pm 0.3$ & $9.6 \pm 0.6$ & $11.4 \pm 0.3$ \\
\hline Rhizopus sp & $9.5 \pm 0.5$ & $10.4 \pm 0.8$ & $11.2 \pm 0.5$ \\
\hline
\end{tabular}


Table 2 Antimicrobial activity of standard antibiotics

\begin{tabular}{|c|c|c|c|c|}
\hline \multirow{2}{*}{ Microorganisms } & \multicolumn{4}{|c|}{ Antibiotics $(\mu \mathrm{g} /$ disc $) /$ Zone of inhibition $(\mathrm{mm})$} \\
\hline & Ofloxacin $(5 \mu \mathrm{g})$ & Tobramycin $(10 \mu \mathrm{g})$ & Gentamycin $(30 \mu \mathrm{g})$ & Ciprofloxacin $(30 \mu \mathrm{g})$ \\
\hline E. coli & 11 & 15 & 7 & 14 \\
\hline S. aureus & 9 & 11 & 7 & 12 \\
\hline K. pneumoniae & 12 & 13 & 9 & 15 \\
\hline P. aerugionosa & 7 & 9 & 6 & 9 \\
\hline S. typhi & 8 & 11 & 9 & 8 \\
\hline
\end{tabular}

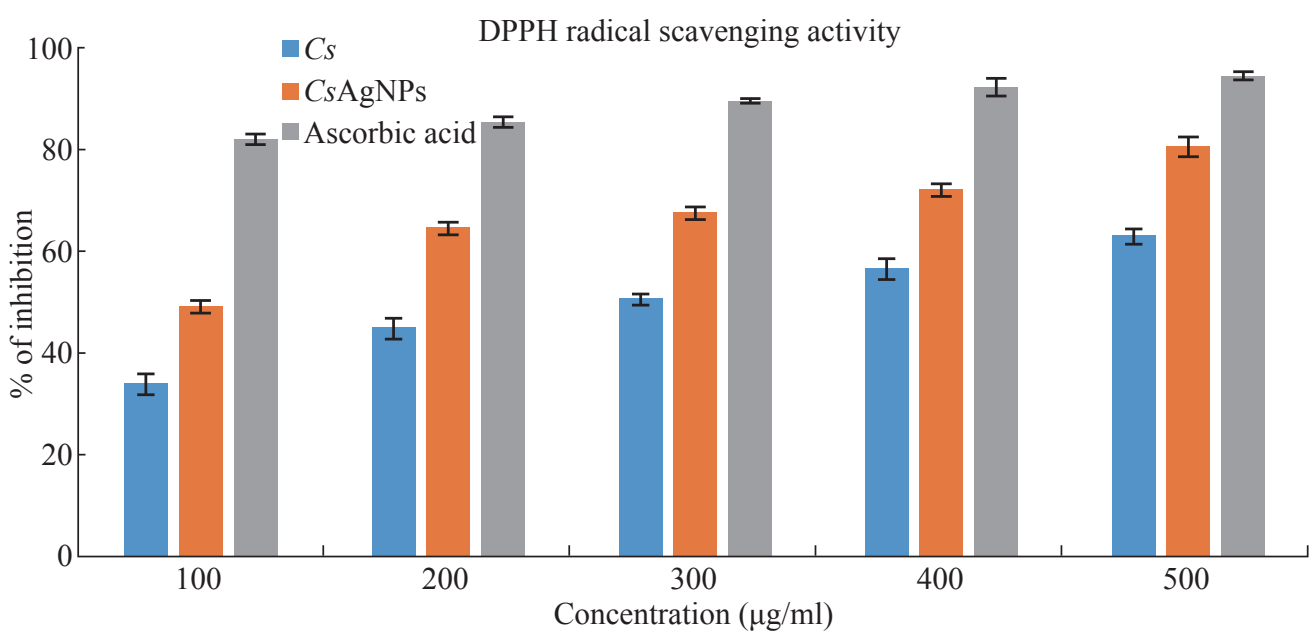

Fig. 8 The reducing power assay of the $C s A g N P s, C s$ aqueous leaf extract and ascorbic acid.

bacterial pathogens were resistant to all five antibiotics (positive control) tested in the study. Among Gram negative bacteria, $K$. pneumoniae was not only strongly inhibited by $C s \mathrm{AgNPs}$ at higher concentration $(150 \mu \mathrm{l})$, but also at lower concentration $(75 \mu \mathrm{l})$ too. Similarly, the growth of $E$. coli was inhibited with zone of inhibition of $14.3 \pm 0.3 \mathrm{~mm}$ at $150 \mu \mathrm{l}$. In case of gram positive bacteria, growth of $S$. aureus was inhibited at $150 \mu \mathrm{l}$ with zone of inhibition of $11.2 \pm 0.8$ $\mathrm{mm}$. Moreover, the antifungal activity of CsAgNPs exhibited higher zone of inhibition of $14.2 \pm 0.3 \mathrm{~mm}$ $(150 \mu \mathrm{l})$ against $C$. albicans. The present study clearly indicates that CsAgNPs exhibited strong antibacterial and antifungal activities against all the clinical isolates even at lower concentration $(75 \mu 1)$. The zone of inhibition was accurately measured and compared with standard antibiotics (Table 2).

Antimicrobial activity of $C$. sulphureus exhibited higher zone of inhibition and increased linearly with the increased concentration of the test sample. The nanoparticle synthesis through biogenic technology exhausted high toxicity towards several bacterial and fungal pathogens like Aeromonas hydrophila, P. fluorescens, Flavobacterium branchiophilum, B. cereus, S. aureus, C. albicans, C. kefyr and A. niger $[1,38]$. In the present work, $C$. sulphureus exhibited better antifungal activities against Fusarium sp. $C$. albicans, Rhizopus sp. whereas Yousefzadi et al. [39] reported that antimicrobial activity of AgNPs were moderate for fungi when compared to bacteria. The reason may due to attachment of AgNPs on the surface of cell wall membrane and disturbs its control function such as permeability and respiration [40]. The silver nanoparticles could be used as an alternative to orthodox antibiotics in the treatment of infections caused by the microorganisms, because they frequently develop resistance to known antibiotics [41]. Phenols and polyphenolic compounds, such as flavonoids, are widely found in food products derived from plant sources, and they have been shown to possess significant antioxidant activities [42]. DPPH stable free radical method is an easy, rapid and sensitive way to survey the antioxidant activity of a specific compound or plant extract [43].

\section{In-vitro antioxidant activity}

Positive DPPH tests demonstrated that $C s$ and 
Cs AgNPs are free radical scavengers. The DPPH scavenging assay exhibited less effective inhibition activity of both $C s$ and $C s A g N P s$ when compared with the standard, ascorbic acid (Fig. 8). The DPPH activity of nanoparticles was found to increase in a dosedependent manner. However, the CsAgNPs exhibited more inhibition with more than $60 \%$ scavenging activity of DPPH than Cs. Cs exhibited potent scavenging effects against $\mathrm{DPPH}$ with an $\mathrm{IC}_{50}$ value of $135.53 \pm 1.78 \mu \mathrm{g} / \mathrm{ml}$ and $C s A g N P s$ showed $\mathrm{IC}_{50}$ value of $92.38 \pm 1.93 \mu \mathrm{g} / \mathrm{ml}$. The $\mathrm{IC}_{50}$ value of standard ascorbic acid for this assay was $60.78 \pm 1.79 \mu \mathrm{g} /$ $\mathrm{ml}$. The free radical DPPH possesses a characteristic absorption at $517 \mathrm{~nm}$ (purple in color), which decreases significantly when exposed to radical-scavengers (due to hydrogen atoms transfer from antioxidant to DPPH). A lower absorbance at $517 \mathrm{~nm}$ indicates a higher radical scavenging activity of extract [44]. The results showed that the ability of $C s$ and $C s A g N P s$ would act as donors of hydrogen atoms or electrons in transformation of DPPH radical into its reduced form of DPPH. Cs AgNPs showed more scavenging activity against DPPH than $C s$ due to its high reduction ability of electrons.

\section{Conclusions}

The biogenic synthesis of silver nanoparticles using aqueous extract of $C$. sulphureus exhibited better antimicrobial activity and could be used in medicinal field as silver dressing of wounds, pharmaceutical nanoengineering for delivering therapeutic agents and in sensors for disease diagnostics. Nowadays, silver nanoparticles are useful as potent antioxidant and anticancer agents. In future, mechanism behind antimicrobial, antioxidant activity $C s$ AgNPs need to be studied precisely.

\section{Acknowledgement}

The author(s) acknowledge the facilities provided by Department of Biotechnology, Karunya University and also grateful to Chancellor (Dr. Paul Dhinakaran), Vice Chancellor (Dr. Sundar Manoharan) and Registrar (Dr. Joseph Kennady), Karunya University, Coimbatore, India for their kind support to carry out this publication.

\section{References}

[1] P.P.N.K. Vijay, S.V.N. Pammib, K. Pratap, et al., Green synthesis and characterization of silver nanoparticles using Boerhaavia diffusa plant extract and their antibacterial activity, Ind. Crops Prod., 2014, 52: 562566.

[2] K.V. Sharma, A.R. Yngard, Y. Lin. Silver nanoparticles green synthesis and their antimicrobial activities, $A d v$. Coll. Interface Sci., 2009, 145: 83-96.

[3] N. Vigneshwaran, A.A. Kathe, P.V. Varadarajan, et al., Functional finishing of cotton fabrics using silver nanoparticles, J. Nanosci. Nanotechnol., 2007, 7: 893897.

[4] Q. Chaudhry, L. Castle. Food applications of nanotechnologies: an overview of opportunities and challenges for developing countries, Trends Food Sci. Technol., 2011, 22: 595-603.

[5] R. Nair, S.H. Varghese, B.G. Nair, et al., Nanoparticulate material delivery to plants, Plant Sci., 2010, 179: 154163.

[6] F.M. Kelly, J.H. Johnston. Colored and functional silver nanoparticle wool fiber composites, Appl. Mater. Interfaces, 2011, 3: 1083-1092.

[7] T.A. Dankovich, D.G. Gray. Bactericidal paper impregnated with silver nanoparticles for point-of-use water treatment, Environ. Sci. Technol., 2011, 45: 19921998.

[8] F. Martinez-Gutierrez, P.L. Olive, A. Banuelos, et al., Synthesis, characterization and evaluation of antimicrobial and cytotoxicity effect of silver and titanium nanoparticles, Nanomed., 2010, 6: 681-688.

[9] D. Yang, S. Chen, P. Huang, et al., Bacteria-template synthesized silver microspheres with hollow and porous structures as excellent SERS substrate, Green Chem., 2010, 12: 2038-2042.

[10] S. Chen, P. Huang, Z. Wang, et al., Self-assembly of gold nanoparticles to silver microspheres as highly efficient 3D SERS substrates, Nanoscale Res. Lett., 2013, 8: 168-174.

[11] J. Virkutyte, R.S. Varma. Green synthesis of metal nanoparticles: biodegradable polymers and enzymes in stabilization and surface functionalization, Chem. Sci., 2011, 2: 837-846.

[12] I. Sivash. Green synthesis of metal nanoparticles using plants, Green Chem., 2011, 13: 2638-2650.

[13] R. Varshney, S. Bhadauria, M.S. Gaur. A review: Biological synthesis of silver and copper nanoparticles, Nano Biomed. Eng. 2012, 4: 99-106.

[14] B.J. Umesh, A.B. Vishwas. Green synthesis of silver nanoparticles using Artocarpus heterophyllus Lam. Seed extract and its antibacterial activity, Ind. Crops Prod., 2013, 46: 132-137.

[15] K. Sunita, N. Sangita, D. Sucheta. Utilization of Cosmos sulphureus Cav. flower dye on wool using mordant combinations, Nat. Prod. Rad., 1999, 5: 19-24.

[16] R. Bindurani, M. Mahesh, K. Kamalesh. Antimicrobial studies on Cosmos sulphureus flowers, Int. J. Pharm. Res. Develop., 2013, 5: 27-31.

[17] A.R. Kamlesh, J.S. Sampada, A.V. Rishikesh, et al., Synthesis and Biological Evaluation of Amino acid Derivatives of Salicylic Acid As Analgesic and Antiinflammatory Agents, Am. J. Pharm. Technol. Res., 2013, 3: $613-620$

[18] K.M. Kumar, B.K. Mandal, M. Sinha, et al., Terminalia chebula mediated green and rapid synthesis of gold nanoparticles, Spectrochimica Acta Part A: Mol. Biomol. Spectro., 2012, 86: 490-494.

[19] C. Perez, M. Paul, P. Bazerque. An antibiotic assay by the agar well diffusion method, Acta Biologiae et Medicinal Experimentalis, 1990, 15: 113-115.

[20] C.W. Chang, K.C. Sei, H.S. Soon, et al., Antioxidant activity and free radical scavenging capacity between Korean medicinal plants and flavonoids by assay-guided 
comparison, Plant Sci., 2002, 163: 1161-1168.

[21] S. Prabhu, E.K. Poulose, Silver nanoparticles: mechanism of antimicrobial action, synthesis, medical applications, and toxicity effects, Int. Nano Lett., 2012, 2: 32.

[22] A.K. Jha, K. Prasad, K. Prasad, et al., Plant system: nature's nanofactory, Colloids Surf. B Biointerfaces, 2009, 73: 219-223.

[23] A.R.V. Nestor, V.S. Mendieta, M.A.C Lopez, et al., Solventless synthesis and optical properties of $\mathrm{Au}$ and $\mathrm{Ag}$ nanoparticles using Camiellia sinensis extract, Mat Lett., 2008, 62: 3103-3105.

[24] A. Rashmi, A. Grewal. Biogenic synthesis, characterization of silver nanoparticles from Candelula officinalis extract and evaluation of their antimicrobial activity, Nano Biomed. Eng. 2015, 7: 47-51.

[25] M. Ya-yuan, T. Yan-kui, W. Sheng-ye, et al., Green synthesis of silver nanoparticles using eucalyptus leaf extract, Mat Lett., 2015, 144: 165-167.

[26] P. Stefano, H. Veera, P. Polina. Biogenic synthesis of antimicrobial silver nanoparticles capped with 1-cysteine, Coll Surf A: Physicochem. Engg. Aspects., 2014, 460: 219-224

[27] M. Kaszuba, D. McKnight, M.T. Connah, et al., Measuring sub nanometre sizes using dynamic light scattering, J. Nanopart. Res., 2008, 10: 823-829.

[28] B. Javad, N. Farideh, R. Tayebe, et al., Green Synthesis of Silver Nanoparticles using Achillea biebersteinii Flower Extract and Its Anti-Angiogenic Properties in the Rat Aortic Ring Model, Mol., 2014, 19: 4624-4634.

[29] B. Ajithaa, K.R.Y. Ashok, R.P. Sreedhara. Green synthesis and characterization of silver nanoparticles using Lantana camara leaf extract, Mat. Sci. Engg C., 2015, 49: 373381.

[30] R. Parameshwaran, S. Kalaiselvam, R. Jayavel. Green synthesis of silver nanoparticles using Beta vulgaris: Role of process conditions on size distribution and surface structure, Mat. Chem. Phy., 2013, 140: 135-147.

[31] V. Marimuthu, K.S. Palanisamy, S. Sesurajan, et al., Biogenic Silver Nanoparticles by Gelidiella acerosa Extract and their Antifungal Effects, Avicenna $J$. Med. Biotechnol., 2011, 3: 143-148.

[32] S. Ponarulselvam, C. Panneerselvam, K. Murugan, et al., Synthesis of silver nanoparticles using leaves of Catharanthus roseus Linn. G. Don and their antiplasmodial activities, Asian Pac. J. Trop. Biomed., 2012, 2: 574-580.

[33] P. Magudapathy, P. Gangopadhyay, B.K. Panigrahi, et al., Electrical transport studies of Ag nanoclusters embedded in glass matrix, Physica B., 2001, 299: 142-146.

[34] S.M. Roopan, Rohit, G. Madhumitha, et al., Low-cost and eco-friendly phyto-synthesis of silver nanoparticles using Cocos nucifera coir extract and its larvicidal activity, Ind.
Crops Prod., 2013, 43: 631-635.

[35] P. Senthil Kumar, S. Sudha. Biosynthesis of silver nanoparticles from Dictyota bartayresiana extract and their antifungal activity, Nano Biomed. Eng. 2013, 5: 7275.

[36] Y. Pulicherla, S. Nataru. Leaf assisted green synthesis of silver nanoparticles from Syzygium alternifolium (wt.) walp. Characterization and antimicrobial studies, Nano Biomed. Eng. 2015, 7: 29-37.

[37] C. Dipankar, S. Murugan. The green synthesis, characterization and evaluation of the biological activities of silver nanoparticles synthesized from Iresine herbstii leaf aqueous extracts, Coll. Surf B-Biointerfaces., 2012, 98: 112-119.

[38] E. David, E.K. Elumalai, T.N.V.K.V. Prasad, et al., Green synthesis of silver nanoparticle using Euphorbia hirta L and their antifungal activities, Arch. Appl. Sci. Res., 2010, 2: 76-81.

[39] M. Yousefzadia, Z. Rahimib, V. Ghafori. The green synthesis, characterization and antimicrobial activities of silver nanoparticles synthesized from green alga Enteromorpha flexuosa (wulfen), J. Agardh. Mat. Lett., 2014, 137: 1-4.

[40] J. Suriya, R.S. Bharathi, V. Sekar, et al., Biosynthesis of silver nanoparticles and its antibacterial activity using seaweed Urospora sp, Afr. J. Biotechnol., 2012, 11: 12192-12198.

[41] V.L. Singleton, R. Orthofer, R.M. Lamuela-Raventos. Analysis of total phenols and other oxidation substrates and antioxidants by means of Folin-Ciocalteu reagent, Methods Enzymol., 1999, 29: 152-178.

[42] S.M. Nabavi, M.A. Ebrahimzadeh, S.F. Nabavi, et al., In vitro Antioxidant and Free radical scavenging activity of Diospyros lotus and Pyrus boissieriana growing in Iran, Pharmacog. Mag., 2009, 4: 122-126.

[43] I.I. Koleva, T.A. Van Beek, J.P.H. Linssen, et al., Screening of plant extracts for antioxidant activity: a comparative study on three testing methods, Phytochem. Anal., 2002, 13: 8-17.

[44] C. Sanchez-Moreno. Review: Methods used to evaluate the free radical scavenging activity in foods and biological systems, Food Sci. Technol. Int., 2002, 28: 121-137.

Copyright $(2016$ Rajkumari Malaka, Jerusha A Hema, Narayanan P Muthukumarasamy, Akilandeswari Sambandam, Subakanmani Subramanian and Murugan Sevanan. This is an open-access article distributed under the terms of the Creative Commons Attribution License, which permits unrestricted use, distribution, and reproduction in any medium, provided the original author and source are credited. 